OCHA-SP-00-06, hep-th/0012058

\title{
Spin chains from super-models
}

\author{
Tetsuo Deguchi* and Pijush K. Ghosh** \\ Department of Physics, Ochanomizu University, \\ 2-1-1 Ohtsuka, Bunkyo-ku, Tokyo 112-8610.
}

\begin{abstract}
We construct and study a class of $N$ particle supersymmetric Hamiltonians with nearest and next-nearest neighbor inverse-square interaction in one dimension. We show that inhomogeneous XY models in an external non-uniform magnetic field can be obtained from these super-Hamiltonians in a particular limit decoupling the fermionic degrees of freedom from the kinematic ones. We further consider a suitable deformation of these super-models such that inhomogeneous XXZ Hamiltonians in an external non-uniform magnetic field are obtained in the same limit. We show that this deformed Hamiltonian with rational potential is, (i) mapped to a set of free super-oscillators through a similarity transformation and (ii) supersymmetric in terms of a new, nonstandard realization of the supercharge. We construct many exact eigenstates of this Hamiltonian and discuss about the applicability of this technique to other models.
\end{abstract}

\footnotetext{
*E-mail address: deguchi@phys.ocha.ac.jp

**E-mail address: pijush@degway.phys.ocha.ac.jp
} 


\section{INTRODUCTION}

The Calogero-Moser-Sutherland (CMS) system is a class of exactly solvable models in one dimension with each particle interacting with the rest of the particles through a long range inverse-square interaction 11 14. These models have been studied over a period of more than thirty years and have been found to be related, directly or indirectly, to a wide class of physical and mathematical systems starting from condensed matter physics to black holes. One such interesting connection is that the CMS Hamiltonians are related to Random Matrix Theory (RMT) describing Brownian motion model of Dyson [2, [15]. Since the RMT

also plays an important role in describing the level statistics of chaotic systems [16], this connection shows an universal aspect of diverse branches of physics [17.

Recently, a class of new models with long-range inverse-square interaction among the particles has been introduced [18]. However, unlike in CMS, particles in these models interact only with the nearest-neighbor(NN) and the next-nearest-neighbor(NNN). Although one would expect each particle to interact with the rest of the particles for systems with longrange interaction, the motivation for considering such an $\mathrm{NN}$ - variant of the CMS model stems from its close connection with Random Banded Matrix (RBM) theory describing short-range Dyson model [19]. In particular, the norm of the ground-state of this NNvariant of CMS system can be identified with the joint-probability distribution function of the Gaussian RBM theory. As a result, different correlation functions can be calculated exactly from the known results of RBM theory. The short-range Dyson model was introduced to understand the level statistics of pseudo-integrable systems [20] like Aharanov-Bohm billiards [21], three dimensional Anderson model at the metal-insulator transition point [22] and polygonal billiards [19. Thus, as in the case of CMS system, these models play an important role in the universal framework of diverse branches of physics like RBM, pseudo- 
integrable system and short-range Dyson model.

The purpose of this paper is to study the supersymmetric version of this relatively new class of CMS-type models with NN and NNN interactions. In particular, we first construct the supersymmetric version of these models. Using the Jordan-Wigner transformation we find that these super-Hamiltonians indeed describe a class of inverse-square Hamiltonians with internal spin-degrees of freedom. The spin part of the Hamiltonian describes a NN XY model in an external magnetic field with the hopping-coefficient proportional to the inversesquare of the distance between the NN particles. We further show that there exists a natural 'Freezing Limit'(FL) for all of these models with stable classical equilibrium configurations, in which the particle and the spin-degrees of freedom decouple completely. Thus, exact eigenstates of these XY models can be obtained from the corresponding exact states of the super-model.

We show that the rational model of this NN variant of CMS system is mapped to a set of free superoscillators through a similarity transformation. This is true for both $A_{N+1}$ and $B C_{N+1}$ type rational super-models. Using this mapping we are able to obtain many exact eigenstates of the rational super-models from the free super-oscillator basis. In absence of any such mapping, we are able to obtain only the ground-state of the Trigonometric and the Hyperbolic super-models.

We suitably deform the super-model such that the XXZ Hamiltonian is contained in the fermionic part of the model and spin-degrees of freedom can be decoupled from the coordinate degrees one in the FL. We also make sure that the deformed Hamiltonian can be shown to be mapped to free-super oscillators through a similarity transformation for the rational case. The realization of the supersymmetry in the deformed model in terms of the usual representation of the super-charges is lost. However, in terms of a non-standard representation of the supercharge, the supersymmetry is still present in the deformed-model 
with rational potential. We obtain the exact ground-state and the first excited state of this deformed super-Hamiltonian and, hence of the corresponding XXZ model obtained in the FL. Though we are not able to go beyond at this point, it is expected that the relation between the super-model and the free super-oscillator would be helpful in obtaining other excited states and may be even the complete spectrum, in future. We also find the exact groundstate of the deformed model corresponding to the super-model of trigonometric and hyperbolic type.

The plan of the paper is the following. We first briefly review the supersymmetric quantum mechanics with many degrees of freedom in the next section. We then show in Sec. III that the most general super-model with stable classical equilibrium configurations has a natural FL in which the particle degrees of freedom completely decouple from the fermionic degrees of freedom. We also determine restrictions on the superpotential such that the fermionic part of a general super-model describes an XY model. In Sec. III.B, we show that the superpotential of the NN-variant of CMS system satisfies this property and study the corresponding rational super-model in some details. We show the mapping of this model to free superoscillators through a similarity transformation and construct many exact eigenstates. A suitable deformation of the super-model to study the XXZ model in the FL has been considered in Sec. IV. We show the mapping of this deformed Hamiltonian to free superoscillators and construct a few exact eigenstates. Finally in Sec. V., we conclude about our findings and discuss on possible future courses of study. The results concerning Jordan-Wigner transformation are reproduced in Appendix A. In appendix B, a class of quadratic superpotentials which give rise to super-Hamiltonians with XY model in its fermionic part is discussed. The fermionic part of the super-model automatically decouples from the parent model for such choices of quadratic superpotentials. We notice that the supersymmetric phase of such super-models corresponds with the ferromagnetic phase 
of the corresponding XY models. In Appendix C, we discuss about the classical minimum equilibrium configurations of the rational inverse-square models. The Trigonometric and the Hyperbolic inverse-square super-models are introduced and studied in Appendices D and E, respectively. Finally, in Appendix F, we discuss the $O S P(2 \mid 2)$ superalgebra of the general inverse-square rational super-models.

\section{SUSY QM WITH MANY DEGREES OF FREEDOM: BRIEF REVIEW}

The supercharge $Q$ and its conjugate $Q^{\dagger}$ are defined as,

$$
Q=\sum_{i=1}^{N} \psi_{i}^{\dagger} a_{i}, \quad Q^{\dagger}=\sum_{i=1}^{N} \psi_{i} a_{i}^{\dagger}
$$

where the fermionic variables $\psi_{i}$ 's satisfy the Clifford algebra,

$$
\left\{\psi_{i}, \psi_{j}\right\}=0=\left\{\psi_{i}^{\dagger}, \psi_{j}^{\dagger}\right\}, \quad\left\{\psi_{i}, \psi_{j}^{\dagger}\right\}=\delta_{i j}, \quad i, j=1,2, \ldots, N
$$

The operators $a_{i}\left(a_{i}^{\dagger}\right)^{\prime}$ 's are analogous to bosonic annihilation ( creation ) operators. They

are defined in terms of the momentum operators $p_{i}=-i \frac{\partial}{\partial x_{i}}$ and the superpotential $W\left(x_{1}, x_{2}, \ldots, x_{N}\right)$ as,

$$
a_{i}=p_{i}-i W_{i}, \quad a_{i}^{\dagger}=p_{i}+i W_{i}, \quad W_{i}=\frac{\partial W}{\partial x_{i}}
$$

and satisfy the following commutation relations among themselves,

$$
\left[a_{i}, a_{j}\right]=0=\left[a_{i}^{\dagger}, a_{j}^{\dagger}\right],\left[a_{i}, a_{j}^{\dagger}\right]=\left[a_{j}, a_{i}^{\dagger}\right]=2 W_{i j}, \quad W_{i j}=\frac{\partial^{2} W}{\partial x_{i} \partial x_{j}}
$$

Note that, by construction, $W_{i}$ 's satisfy the so called 'zero-curvature condition' $\partial_{i} W_{j}=$ $\partial_{j} W_{i}$. Also, for translationally invariant superpotentials, these $W_{i}$ 's satisfy the 'sum to zero' condition, $\sum_{i} W_{i}=0$. These two properties are useful ingredients in studying the usual CMS model. 
The supersymmetric Hamiltonian is defined in terms of the supercharges as,

$$
\begin{aligned}
H & =\frac{1}{2}\left\{Q, Q^{\dagger}\right\} \\
& =\frac{1}{4} \sum_{i}\left\{a_{i}, a_{i}^{\dagger}\right\}+\frac{1}{4} \sum_{i, j}\left[a_{i}, a_{j}^{\dagger}\right]\left[\psi_{i}^{\dagger}, \psi_{j}\right] .
\end{aligned}
$$

The Hamiltonian commutes with both $Q$ and $Q^{\dagger}$. The ground state of $H$ is annihilated by both $Q$ and $Q^{\dagger}$. Thus, the ground states are given by,

$$
\phi_{0}=e^{-W}\left|0>, \quad \phi_{N}=e^{W}\right| \overline{0}>,
$$

where the fermionic vacuum $\mid 0>$ and its conjugate $\mid \overline{0}>$ in the $2^{N}$ dimensional fermionic Fock space are defined as,

$$
\psi_{i}\left|0>=0, \quad \psi_{i}^{\dagger}\right| \overline{0}>=0
$$

The first equation of (17) defines the zero-fermion sector, while the second one defines the $N$ fermion sector. If either $\phi_{0}$ or $\phi_{N}$ is normalizable, the supersymmetry is preserved with zero ground state energy. On the other hand, the supersymmetry is broken if neither $\phi_{0}$ nor $\phi_{N}$ is normalizable. The ground state energy in this case is positive-definite.

\section{XY MODELS AND SUSY QM WITH NEAREST-NEIGHBOR INTERACTION}

\section{A. General Case}

The supersymmetric Hamiltonian (5) can be written as,

$$
H=\frac{1}{2} \sum_{i}\left(p_{i}^{2}+W_{i}^{2}\right)+\frac{1}{2} \sum_{i, j} W_{i j}\left(\psi_{i}^{\dagger} \psi_{j}-\psi_{i} \psi_{j}^{\dagger}\right) .
$$

Equation (4) has been used to write Eq. (8) in its present form. The term $W_{i j}$ is independent of the bosonic coordinates for the choice of a purely quadratic superpotential, $W=\sum_{i, j} x_{i} S_{i j} x_{j}$ with $S$ any real, symmetric matrix. Thus, the bosonic and the fermionic 
Hamiltonians in (8) decouple automatically from each other. This class of superpotentials producing XY models in the fermionic part have been discussed in Appendix B. For all other nontrivial choices of $W$, however, the bosonic and the fermionic degrees of freedom are coupled together. They can be decoupled only in certain special limits. We find that the FL described in [4] is well suited for the Hamiltonian (8) to decouple the fermionic part from its parent Hamiltonian. In particular, let us assume that $W$ is proportional to an overall coupling constant $\lambda$. Then the coefficient of the bosonic potential becomes $\lambda^{2}$, while it is $\lambda$ for the fermionic part of the Hamiltonian. Now we take the strong interaction limit $\lambda \rightarrow \infty$. This can be achieved by multiplying $H$ with $\lambda^{-2}$ and taking $\lambda \rightarrow \infty$. The leading relevant term in this limit is,

$$
H \equiv \frac{1}{2 \lambda} \sum_{i, j} W_{i j}\left(\psi_{i}^{\dagger} \psi_{j}-\psi_{i} \psi_{j}^{\dagger}\right)+O\left(\lambda^{-2}\right),
$$

where the bosonic coordinates in $W_{i j}$ take the value of their classical minimum equilibrium configurations, $W_{i}=0$. Note that a leading, but non-dynamical and irrelevant term $\sum_{i} W_{i}^{2}$ has not been included in (9), which identically vanishes for the classical minimum equilibrium configurations.

We would now like to see if the superpotential can be chosen such that the fermionic part of $H$ can be identified with some spin chain model in the FL. We choose the superpotential $W$ such that,

$$
W_{i j}=\delta_{i j} g_{i}\left(x_{1}, x_{2}, \ldots, x_{N}\right)+\delta_{i, j+1} h_{i-1}\left(x_{1}, x_{2}, \ldots, x_{N}\right)+\delta_{i, j-1} h_{i}\left(x_{1}, x_{2}, \ldots, x_{N}\right)
$$

where $h_{i}$ 's and $g_{i}$ 's are arbitrary functions of the bosonic coordinates. With this choice of superpotential, the Hamiltonian now reads,

$$
H=\frac{1}{2} \sum_{i}\left(p_{i}^{2}+W_{i}^{2}\right)+\frac{1}{2} \sum_{i}\left[g_{i} n_{i}+2 h_{i}\left(\psi_{i}^{\dagger} \psi_{i+1}-\psi_{i} \psi_{i+1}^{\dagger}\right)\right], \quad n_{i}=\left[\psi_{i}^{\dagger}, \psi_{i}\right]
$$

Note that the $n_{i}$ 's are proportional to the fermionic number operators $\psi_{i}^{\dagger} \psi_{i}$ for each fermionic degree of freedom $\psi_{i}$ and the second term inside the square bracket with the coefficient $h_{i}$ is 
the XY term at site $i$ in the fermionic representation. In particular, using the Jordan-Wigner transformation given in Appendix A with the periodic boundary conditions, we have,

$$
H=\frac{1}{2} \sum_{i}\left(p_{i}^{2}+W_{i}^{2}\right)+\frac{1}{2} \sum_{i}\left[g_{i} \sigma_{i}^{z}+h_{i}\left(\sigma_{i}^{x} \sigma_{i+1}^{x}+\sigma_{i}^{y} \sigma_{i+1}^{y}\right)\right]
$$

Thus, the supersymmetric Hamiltonian $H$, in general, describes an $N$ particle system with both kinematical and internal spin degrees of freedom. Note that both $g_{i}$ 's and $h_{i}$ 's depend on the bosonic coordinates. In the FL, as described above, it is possible to decouple the spin degrees of freedom from the coordinate degrees of freedom. For such cases, solving the supersymmetric Hamiltonian $H$, one would in fact also be able to solve the corresponding spin chain problem. We illustrate this point with a few examples below. We use the Hamiltonian (11) with the fermionic representation for the spin variables for all practical purposes.

A comment is in order. The XY model as the fermionic part of a supersymmetric quantum mechanical model has first been identified in Ref. [23]. However, such identification for an arbitrary superpotential is not correct. The Jordan-Wigner transformation maps a nearest-neighbor XY model to a free fermionic theory with the hopping allowed to the next nearest site only and the vice versa. It is necessary that the superpotential has a form given by Eq. (10) for such fermionic bilinear to be present in a supersymmetric theory. Thus, for the first time in the literature, we give the necessary condition to be satisfied by the superpotential so that nearest-neighbor XY models are contained in the fermionic sector of the supersymmetric theory. Such a criteria is missing in Ref. [23] and, to the best of our knowledge, in all subsequent papers on this subject. We would like to mention here that for a general superpotential which does not satisfy (10), one can still obtain a fermionic theory in the FL. However, the Jordan-Wigner transformation may not be applicable to map such a fermionic theory into a nearest-neighbor XY model. 


\section{B. Hamiltonians with Inverse-square interaction}

In this section, we consider rational super-models with inverse-square interaction. The Trigonometric and the Hyperbolic version of these classes of inverse-square models are discussed in Appendix D and Appendix E, respectively. For the rational model, we choose the super-potential of the following form,

$$
W=-w+\frac{\omega}{2} \sum_{i} x_{i}^{2}, \quad w=\ln G\left(x_{1}, x_{2}, \ldots, x_{N}\right),
$$

where $G$ is a homogeneous function of any positive degree $d$ :

$$
\sum_{i} x_{i} \frac{\partial G}{\partial x_{i}}=d G
$$

The Hamiltonian for this choice of superpotential has a dynamical $O S P(2 \mid 2)$ supersymmetry [9]. The relevant algebra is given in Appendix F. Using the subalgebra $O(2,1) \times U(1)$, one can show that the Hamiltonian is mapped to a set of free superoscillators through a similarity transformation [9]. We use this result very often in this section to construct exact eigenstates. We shall specialize to two different choices of $G$.

A comment regarding the similarity transformation is in order. One might wonder that any super-Hamiltonian with the superpotential $W$ described by (13) and (14) is exactly solvable, due to its mapping to free super oscillators through similarity transformations. We would like to point out that this may not be true always, because, merely mapping the original Hamiltonian to free super-oscillators is not sufficient for such conclusions. We have to make sure that the similarity transformation, which is responsible for such mapping, keeps the original Hamiltonian in its own Hilbert space. Thus, as a check, one should show that the complete spectrum and the corresponding well-behaved, normalizable eigen-functions of $H$ can be constructed from the Hamiltonian of the free super-oscillators through inverse similarity transformation. The formal equivalence at the operator level acts as a necessary 
condition, while the construction of the complete spectrum and associated well-behaved eigen-functions of the original Hamiltonian from the super-oscillator model is sufficient to claim the equivalence between these two Hamiltonians.

\section{Rational model of $A_{N+1}$-type}

We choose the superpotential as,

$$
G=\prod_{i=1}^{N}\left(x_{i}-x_{i+1}\right)^{\lambda}, \quad x_{N+i}=x_{i}
$$

Note that $G$ is a homogeneous function of degree $d=\lambda N$. This produces the following expressions for $g_{i}$ and $h_{i}$,

$$
h_{i}=-\lambda\left(x_{i}-x_{i+1}\right)^{-2}, \quad g_{i}=\omega-\left(h_{i}+h_{i-1}\right) .
$$

The Hamiltonian $H$ for the rational superpotential now reads,

$$
\begin{aligned}
H_{R} & =-\frac{1}{2} \sum_{i} \frac{\partial^{2}}{\partial x_{i}^{2}}+\frac{\lambda^{2}}{2} \sum_{i}\left[2\left(x_{i}-x_{i+1}\right)^{-2}-\left(x_{i-1}-x_{i}\right)^{-1}\left(x_{i}-x_{i+1}\right)^{-1}\right]+\frac{1}{2} \omega^{2} \sum_{i} x_{i}^{2} \\
& -\lambda \omega N+\lambda \sum_{i}\left[\left(x_{i}-x_{i+1}\right)^{-2}\left(\frac{1}{2}\left(n_{i}+n_{i+1}\right)-\psi_{i}^{\dagger} \psi_{i+1}+\psi_{i} \psi_{i+1}^{\dagger}\right)+\frac{\omega}{2 \lambda} n_{i}\right]
\end{aligned}
$$

with the periodic boundary conditions on the fermionic variables: $\psi_{N+i}=\psi_{i}$. This is the supersymmetric generalization of a class of Hamiltonian recently introduced and studied in [18]. Unlike in CMS Hamiltonians [四3], particles in this model interact with each other through the NN and the NNN interaction. Note that the bosonic many-body interaction is of NN as well as NNN-type. On the other hand, the fermionic interaction is only of NN-type. For the special case of $N=3, H_{R}$ reduces to the rational CMS super-Hamiltonian due to the periodic boundary conditions imposed on the bosonic and the fermionic coordinates. We would like to mention here that our whole analysis goes through also for the open boundary conditions. The third term with the coefficient $\lambda$ contains the XY Hamiltonian in terms of 
fermionic variables. Thus, particles in this model are also having internal spin degrees of freedom.

The groundstate of $H$ in the supersymmetric phase $(\lambda>0)$ is given by,

$$
\phi_{0}=\prod_{i}\left(x_{i}-x_{i+1}\right)^{\lambda} e^{-\frac{\omega}{2} \sum_{i} x_{i}^{2}} \mid 0>.
$$

The wavefunction $\phi_{0}$ is normalizable for $\lambda>-\frac{1}{2}$. However, a stronger criteria that each momentum operator $p_{i}$ is self-adjoint for the wavefunction of the form $\phi_{0}$ requires $\lambda>0$. The supersymmetry is preserved for $\lambda>0$, while it is broken for $\lambda<0$. The groundstate of $H$ in the supersymmetry-breaking phase $(\lambda<0)$ is given by,

$$
\tilde{\phi}_{0}=\prod_{i}\left(x_{i}-x_{i+1}\right)^{-\lambda} e^{-\frac{\omega}{2} \sum_{i} x_{i}^{2}} \mid \overline{0}>.
$$

The wavefunction $\tilde{\phi}_{0}$ is normalizable for $\lambda<\frac{1}{2}$. However, the self-adjointness criteria of each momentum operator $p_{i}$ determines $\lambda<0$. The eigenspectrum of $H$ in the supersymmetrybreaking phase can be described by using a 'duality property' of this kind of Hamiltonian [9]. Thus, we consider only the supersymmetric phase of $H$ in this paper. Note that the effect of going from the supersymmetric phase to supersymmetry-breaking phase of $H$ is just an overall change in the sign of the coupling constant for the corresponding spin chain model.

The total fermion number operator $N_{f}=\sum_{i} \psi_{i}^{\dagger} \psi_{i}$ commutes with the Hamiltonian $H_{R}$ and $G$ is a homogeneous function of a fixed degree. Following the treatment of Ref. [9], it can be shown that the Hamiltonian is mapped to free superoscillators through a similarity transformation. In particular,

$$
\begin{aligned}
H_{\text {sho }} & =T H_{R} T^{-1} \\
& =\omega \sum_{i}\left(x_{i} \frac{\partial}{\partial x_{i}}+\psi_{i}^{\dagger} \psi_{i}\right),
\end{aligned}
$$

where the operator $T=e^{\frac{S}{2}} e^{W}$ is given by, 


$$
\begin{aligned}
S & =\frac{1}{2} \sum_{i} \frac{\partial^{2}}{\partial x_{i}^{2}}+\lambda \sum_{i}\left(x_{i}-x_{i+1}\right)^{-1}\left(\frac{\partial}{\partial x_{i}}-\frac{\partial}{\partial x_{i+1}}\right) \\
& -\lambda \sum_{i}\left(x_{i}-x_{i+1}\right)^{-2}\left[\left(\psi_{i}^{\dagger} \psi_{i}+\psi_{i+1}^{\dagger} \psi_{i+1}\right)-\left(\psi_{i}^{\dagger} \psi_{i+1}-\psi_{i} \psi_{i+1}^{\dagger}\right)\right] .
\end{aligned}
$$

The relation (20) opens up the possibility of constructing the eigenstates of $H_{R}$ from that of $H_{\text {sho }}$. In particular, if $P_{n} \mid 0>$ is an eigenfunction of $H_{\text {sho }}$ with the eigenvalue $E_{n}$, then, $H_{R}$ has the same eigenvalue $E_{n}$ with the eigenfunction given by,

$$
\chi=T^{-1} P_{n} \mid 0>
$$

$P_{n}$ in (22), in general, is a function of both the bosonic and the fermionic coordinates. If we choose $P_{n}$ to be a single-particle wave-function of $H_{s h o}, \chi$ contains an essential singularity. This is not physically acceptable. This shows that the original many-body problem is a highly correlated system and we have to choose $P_{n}$ as suitable combinations of all the single-particle states. We find that the following choices of $P_{n}$,

$$
\begin{aligned}
P_{n}^{I}= & \sum_{i} x_{i}^{n} \psi_{i}^{\dagger}, \quad P_{n}^{I I}=\sum_{i \neq j}\left(x_{i}^{n}-x_{j}^{n}\right) \psi_{i}^{\dagger} \psi_{j}^{\dagger}, \quad n=0,1,2,3, \\
P^{I I I}= & \sum_{i \neq j \neq k} \operatorname{Det}\left(\begin{array}{ccc}
1 & 1 & 1 \\
x_{i}^{m} & x_{j}^{m} & x_{k}^{m} \\
x_{i}^{n} & x_{j}^{n} & x_{k}^{n}
\end{array}\right) \psi_{i}^{\dagger} \psi_{j}^{\dagger} \psi_{k}^{\dagger}, \quad m \neq n, \quad m, n=1,2,3, \\
P^{I V}= & \sum_{i \neq j \neq k \neq l} \operatorname{Det}\left(\begin{array}{cccc}
1 & 1 & 1 & 1 \\
x_{i} & x_{j} & x_{k} & x_{l} \\
x_{i}^{2} & x_{j}^{2} & x_{k}^{2} & x_{l}^{2} \\
x_{i}^{3} & x_{j}^{3} & x_{k}^{3} & x_{l}^{3}
\end{array}\right) \psi_{i}^{\dagger} \psi_{j}^{\dagger} \psi_{k}^{\dagger} \psi_{l}^{\dagger},
\end{aligned}
$$

result in well-behaved, non-singular $\chi$. Unlike in the case of super-CMS system, there are no compelling reasons to consider only those $P_{n}$ which are symmetric under the combined exchange of the bosonic and the fermionic coordinates. Surprisingly, it turns out that the $P_{n}$ corresponding to the exact eigenstates $\chi$ found by us necessarily have this property. This 
can be checked easily for $P_{n}^{I}$ and $P_{n}^{I I}$. It may be worth mentioning here that the matrices appearing in $P_{I I I}$ and $P_{I V}$ above are the Slater determinants constructed out of the singleparticle bosonic wave-functions. As a result, they are antisymmetric under the exchange of any two indices. Together with the antisymmetric nature of the fermionic variables, it is easy to see that $P_{I I I}$ and $P_{I V}$ are invariant under the combined exchange of the bosonic and the fermionic coordinates. Note that unlike in the rational super-CMS system, the maximum power which a single-particle coordinate $x_{i}$ can have in $P_{n}^{I}, P_{n}^{I I}, P^{I I I}$ and $P^{I V}$, is required to be 3 . The construction of $P^{k}$ as a Slater determinant in terms of single-particle bosonic states for $k \geq 5$ necessarily involves a single-particle coordinate with power $n \geq 4$. Thus, we find all $P^{k}$ for $k \geq 5$ produces singular $\chi$ for the model under consideration. It may be recalled here that the similar construction of $P^{k}$ with $k$ ranging from 1 to $N$ produces well-behaved $\chi$ for the rational super-CMS system.

In equation (23), the $P$ 's correspond to 'basic excitations' with $N_{f}=1,2,3$ and 4 . One can construct many other $P$ producing non-singular $\chi$ by suitably combining these four $P$ 's and the symmetric polynomials,

$$
r_{n, m}=\left(\sum_{i} x_{i}^{n}\right)^{m}, \quad n=1,2,3 ; m=1,2, \ldots, \infty
$$

For example, the choice $P=r_{n, m} P_{k}^{I} P_{l}^{I I}$ produces non-singular $\chi$. We now give an algebraic construction of the spectrum. Consider the following set of operators,

$$
\begin{aligned}
& b_{i}^{-}=i p_{i}=\frac{\partial}{\partial x_{i}}, \quad b_{i}^{+}=2 \omega x_{i} \\
& B_{n}^{-}=\sum_{i=1}^{N} T^{-1} b_{i}^{-n} T, \quad B_{n}^{+}=\sum_{i=1}^{N} T^{-1} b_{i}^{+^{n}} T, \quad n=1,2,3 \\
& F_{m}^{-}=\sum_{i} T^{-1} \psi_{i} b_{i}^{-{ }^{m-1}} T, \quad F_{m}^{+}=\sum_{i} T^{-1} \psi_{i}^{\dagger} b_{i}^{+^{m-1}} T, \quad m=1,2,3,4, \\
& q_{n}^{-}=T^{-1} \sum_{i} \psi_{i}^{\dagger} b_{i}^{-n} T, \quad q_{n}^{+}=T^{-1} \sum_{i} \psi_{i} b_{i}^{+^{n}} T, \quad n=1,2,3 .
\end{aligned}
$$

Note that there are three bosonic anhilation(creation) operators $B_{n}^{-}\left(B_{n}^{+}\right)$and four fermionic 
anhilation(creation) operators $F_{n}^{-}\left(F_{n}^{+}\right)$. This is because the highest power of $b_{i}^{+}$should be 3. These operators satisfy the following algebra,

$$
\begin{aligned}
& \left\{F_{m}^{+}, F_{n}^{+}\right\}=0, \quad\left[B_{m}^{+}, F_{n}^{+}\right]=0, \quad\left[B_{m}^{+}, B_{n}^{+}\right]=0, \\
& \left\{q_{1}^{-}, F_{n}^{+}\right\}=0, \quad\left\{q_{1}^{+}, F_{n}^{+}\right\}=B_{n}^{+}, \quad\left[H, F_{n}^{+}\right]=n \omega F_{n}^{+}, \\
& {\left[q_{1}^{-}, B_{n}^{+}\right]=2 n \omega F_{n}^{+}, \quad\left[q_{1}^{+}, B_{n}^{+}\right]=0, \quad\left[H, B_{n}^{+}\right]=n \omega B_{n}^{+} .}
\end{aligned}
$$

The eigenstates can now be created in an algebraic manner by using the above relations. In particular,

$$
\chi_{n_{1} \ldots n_{3} \nu_{1} \ldots \nu_{4}}=\prod_{k=1}^{3} B_{k}^{+^{n_{k}}} F_{k}^{+\nu_{k}} F_{4}^{+^{\nu_{4}}} \phi_{0},
$$

is the eigenfunction with the eigen-value,

$$
E=\omega\left(\sum_{k=1}^{3} k\left(n_{k}+\nu_{k}\right)+4 \nu_{4}\right) .
$$

The bosonic quantum numbers $n_{k}$ 's are nonnegative integers, while the fermionic quantum numbers $\nu_{k}$ 's are either 0 or 1 . Note that a system consisting of a set of three independent super-oscillators with the frequencies $\omega, 2 \omega, 3 \omega$ and a fermionic oscillator with the frequency $4 \omega$ have the same energy $E$. Thus, the spectrum of the model is identical to that of a system consisting of three independent super-oscillators with the frequencies $\omega, 2 \omega, 3 \omega$ and a fermionic oscillator with the frequency $4 \omega$. The physical reason behind the disparity between the bosonic and the fermionic excitations deserves further understandings.

In order to take the FL described in [4], we first scale $\omega$ as $\lambda \omega$ and then take the limit $\lambda \rightarrow \infty$. The spin degrees of freedom decouple completely from the kinematical ones in this strong interaction limit. The dynamics of the system is lost and the particles are frozen at their classical equilibrium configurations,

$$
W_{i}=\lambda \omega x_{i}+\lambda\left[\left(x_{i-1}-x_{i}\right)^{-1}-\left(x_{i}-x_{i+1}\right)^{-1}\right]=0 .
$$


In particular, we multiply the Hamiltonian $H$ with $\lambda^{-2}$ and take the limit $\lambda \rightarrow \infty$. The relevant leading term in this limit gives an XY Hamiltonian,

$$
H_{s}=\sum_{i}\left[\left(x_{i}-x_{i+1}\right)^{-2}\left(\frac{1}{2}\left(n_{i}+n_{i+1}\right)-\psi_{i}^{\dagger} \psi_{i+1}+\psi_{i} \psi_{i+1}^{\dagger}\right)+\frac{\omega}{2} n_{i}\right]
$$

where $x_{i}^{\prime} s$ are determined from (29). We are not able to solve (29) for arbitrary $N$. However, we show in Appendix C that the equilibrium configurations of the particles necessarily constitute a non-uniform lattice.

\section{Rational model of $B C_{N+1}$-type}

We choose the superpotential as,

$$
G\left(\lambda, \lambda_{1}, \lambda_{2}\right)=\prod_{i}\left(x_{i}^{2}-x_{i+1}^{2}\right)^{\lambda} \prod_{j} x_{j}^{\lambda_{1}} \prod_{k}\left(2 x_{k}\right)^{\lambda_{2}},
$$

where $\lambda, \lambda_{1}$ and $\lambda_{2}$ are arbitrary parameters. The $D_{N+1}$-type model is described by taking $\lambda_{1}=\lambda_{2}=0$, while the case $\lambda_{1}=0\left(\lambda_{2}=0\right)$ describes $C_{N+1}\left(B_{N+1}\right)$-type model. We restrict ourselves to the $B_{N+1}$-type Hamiltonian, without loss of any generality. We introduce two new variables as,

$$
q_{i}=x_{i}-x_{i+1}, \quad \bar{q}_{i}=x_{i}+x_{i+1}
$$

The Hamiltonian $H$ in terms of these new variables is given by,

$$
\begin{aligned}
H & =\frac{1}{2} \sum_{i} p_{i}^{2}+\lambda^{2} \sum_{i}\left[q_{i}^{-2}+\bar{q}_{i}^{-2}-\left(q_{i-1}^{-1}-\bar{q}_{i-1}^{-1}\right)\left(q_{i}^{-1}+\bar{q}_{i}^{-1}\right)\right]+\frac{1}{2} \sum_{i}\left(\omega^{2} x_{i}^{2}+\frac{\lambda_{1}}{x_{i}^{2}}\right) \\
& -\lambda \omega\left(2+\frac{\lambda_{1}}{\lambda}\right) N+\frac{\lambda}{2} \sum_{i} q_{i}^{-2}\left[\left(n_{i}+n_{i+1}\right)-2\left(\psi_{i}^{\dagger} \psi_{i+1}-\psi_{i} \psi_{i+1}^{\dagger}\right)\right] \\
& +\frac{\lambda}{2} \sum_{i} \bar{q}_{i}^{-2}\left[\left(n_{i}+n_{i+1}\right)+2\left(\psi_{i}^{\dagger} \psi_{i+1}-\psi_{i} \psi_{i+1}^{\dagger}\right)\right]+\sum_{i} \frac{1}{2}\left(\omega+\frac{\lambda_{1}}{x_{i}^{2}}\right) n_{i} .
\end{aligned}
$$

The ground-state in the supersymmetry-preserving phase $\left(\lambda>0, \lambda_{1}>0\right)$ is given by, 


$$
\psi_{0}=\prod_{i}\left(x_{i}^{2}-x_{i+1}^{2}\right)^{\lambda} \prod_{j} x_{j}^{\lambda_{1}} e^{-\frac{\omega}{2} \sum_{i} x_{i}^{2}} \mid 0>.
$$

On the other hand, in the supersymmetry breaking phase, the groundstate has the following expressions,

$$
\begin{aligned}
& \psi_{0}^{I}=\prod_{i}\left(x_{i}^{2}-x_{i+1}^{2}\right)^{-\lambda} \prod_{j} x_{j}^{-\lambda_{1}} e^{-\frac{\omega}{2} \sum_{i} x_{i}^{2} \mid \overline{0}>, \quad \lambda, \lambda_{1}<0,} \\
& \psi_{0}^{I I}=\prod_{i}\left(x_{i}^{2}-x_{i+1}^{2}\right)^{-\lambda} \prod_{j} x_{j}^{1+\lambda_{1}} e^{-\frac{\omega}{2} \sum_{i} x_{i}^{2}} \mid \overline{0}>, \quad \lambda<0, \lambda_{1}>0 .
\end{aligned}
$$

We do not know the groundstate in the region $\lambda>0, \lambda_{1}<0$. This is partially because the known method [9] uses the 'shape-invariance' property of the many-body potential to determine the groundstate in such supersymmetry breaking phases. Unfortunately, the manybody potential in the present case is not 'shape invariant' due to the presence of the threebody term. It might be recalled here that in the case of $B C_{N+1}$ type super-CMS, there is no three-body term and the many-body potential is 'shape invariant' [9,11]. Thus, the groundstate of super-CMS of $B C_{N+1}$ type can be obtained in all the regions of the parameter space.

We study only the supersymmetric phase of $H$ in this section and construct some exact eigenstates. Following [9], similar construction of exact eigenstates in the two supersymmetry-breaking phases associated with the groundstates $\psi_{0}^{I}$ and $\psi_{0}^{I I}$ can be carried over in a straightforward way.

The Hamiltonian (33) can be mapped to a set of free superoscillators through a similarity transformation, $H_{\text {sho }}=\mathcal{T} H \mathcal{T}^{-1}$, where the $W$ in $\mathcal{T}=e^{\frac{\hat{s}}{2}} e^{W}$ is determined from (31) and $\hat{S}$ is given by,

$$
\begin{aligned}
\hat{S} & =\sum_{i}\left[-\frac{1}{2} p_{i}^{2}+\lambda\left(q_{i}^{-1}+\bar{q}_{i}^{-1}+\frac{\lambda_{1}}{\lambda} x_{i}^{-1}\right)\left(\frac{\partial}{\partial x_{i}}-\frac{\partial}{\partial x_{i+1}}\right)\right]-\lambda \sum_{i} q_{i}^{-2}\left(\psi_{i}^{\dagger} \psi_{i}+\psi_{i+1}^{\dagger} \psi_{i+1}\right) \\
& +\lambda \sum_{i} q_{i}^{-2}\left(\psi_{i}^{\dagger} \psi_{i+1}-\psi_{i} \psi_{i+1}^{\dagger}\right)+\lambda \sum_{i} \bar{q}_{i}^{-2}\left[\left(\psi_{i}^{\dagger} \psi_{i}+\psi_{i+1}^{\dagger} \psi_{i+1}\right)+\left(\psi_{i}^{\dagger} \psi_{i+1}-\psi_{i} \psi_{i+1}^{\dagger}\right)\right] \\
& +\sum_{i} \frac{\lambda_{1}}{2} x_{i}^{-2} n_{i} .
\end{aligned}
$$


We find that the physically acceptable wave-function $\chi=\mathcal{T}^{-1} P \mid 0>$ of $H$ can be obtained for the following choices of $P$,

$$
\begin{aligned}
& P_{n}^{I}=\sum_{i} x_{i}^{2 n}\left(x_{i} \psi_{i}^{\dagger}\right), n=0,1, \\
& P^{I I}=\sum_{i \neq j}\left(x_{i}^{2}-x_{j}^{2}\right)\left(x_{i} \psi_{i}^{\dagger}\right)\left(x_{j} \psi_{j}^{\dagger}\right) .
\end{aligned}
$$

Note that both $P^{I}$ and $P^{I I}$ are invariant under the following two transformations, (i) $\left(x_{i}, \psi_{i}^{\dagger}\right) \leftrightarrow\left(x_{j}, \psi_{j}^{\dagger}\right)$ and (ii) $\left(x_{i}, \psi_{i}^{\dagger}\right) \rightarrow\left(-x_{i},-\psi_{i}^{\dagger}\right)$. These are also the symmetry of the corresponding expression for the $B C_{N+1}$-type rational super-CMS [9]. We are unable to find any other $P$ which produces nonsingular $\chi$. Note that the maximum power of a singleparticle coordinate is given by 3 in both $P_{I}$ and $P_{I I}$. Any attempt to construct other $P$ with higher fermion numbers and having the properties (i) and (ii) necessarily involves a single-particle coordinate with power greater than three. This produces singular $\chi$.

The eigen-spectrum also can be constructed in an algebraic way. We define the following set of creation and annihilation operators,

$$
\mathcal{B}_{1}^{+}=\mathcal{T}^{-1} \sum_{i} b_{i}^{+2} \mathcal{T}, \quad \mathcal{F}_{n}^{+}=\mathcal{T}^{-1} \sum_{i} \psi_{i}^{\dagger} b_{i}^{+^{2 n-1}} \mathcal{T}, \quad n=1,2
$$

These operators satisfy an algebra similar to (26). Note there are only one bosonic operator, while two are fermionic operators. This is again related to the fact that the highest power of $b_{i}^{\dagger}$ should be 3 . The eigen-states are obtained as,

$$
\chi_{n_{1}, \nu_{1}, \nu_{2}}=\mathcal{B}_{1}^{+{ }^{n_{1}}} \mathcal{F}_{1}^{+^{\nu_{1}}} \mathcal{F}_{2}^{+^{\nu_{2}}} \phi_{0},
$$

with the energy $\mathcal{E}=2 \omega\left(n_{1}+\nu_{1}\right)+4 \omega \nu_{2}$. The bosonic quantum number is non-negative integers, while the fermionic quantum numbers are 0 or 1 . The spectrum is that of a superoscillator with the frequency $2 \omega$ and a fermionic oscillator with the frequency $4 \omega$. 


\section{XXZ MODEL}

It is known that the XXZ model describes an interacting fermionic theory with the interaction term proportional to $\sum_{i} n_{i} n_{i+1}$, which is quartic in the fermionic variables. The super-Hamiltonian $H$ being at most quadratic in these variables, our methods described in the previous section should be modified to make room for the study of XXZ model. We deform the supersymmetric Hamiltonian $H$ to $\mathcal{H}$ with the deformation parameter $\delta$,

$$
\begin{aligned}
\mathcal{H} & =H+2 \delta \sum_{i} h_{i} \psi_{i}^{\dagger} \psi_{i} \psi_{i+1}^{\dagger} \psi_{i+1} \\
& =\frac{1}{2} \sum_{i}\left(p_{i}^{2}+W_{i}^{2}\right)+\frac{1}{2} \sum_{i}\left[g_{i} n_{i}+2 h_{i}\left(\psi_{i}^{\dagger} \psi_{i+1}-\psi_{i} \psi_{i+1}^{\dagger}+2 \delta \psi_{i}^{\dagger} \psi_{i} \psi_{i+1}^{\dagger} \psi_{i+1}\right)\right] .
\end{aligned}
$$

The XXZ Hamiltonian in an external magnetic field is contained in $\mathcal{H}$,

$$
\mathcal{H}=\frac{1}{2} \sum_{i}\left(p_{i}^{2}+W_{i}^{2}\right)+\frac{1}{2} \sum_{i}\left[g_{i} \sigma_{i}^{z}+h_{i}\left(\sigma_{i}^{x} \sigma_{i+1}^{x}+\sigma_{i}^{y} \sigma_{i+1}^{y}+\delta\left(1+\sigma_{i}^{z}\right)\left(1+\sigma_{i+1}^{z}\right)\right)\right] .
$$

With the choice of a suitable $W$, the spin degrees of freedom can be decoupled from the kinematical one in the FL. In the process, we obtain an inhomogeneous XXZ model in an external magnetic field,

$$
H_{x x z}=\frac{1}{2} \sum_{i}\left[\delta h_{i}+\left(g_{i}+\delta h_{i}\right) \sigma_{i}^{z}+\delta h_{i} \sigma_{i+1}^{z}+h_{i}\left(\sigma_{i}^{x} \sigma_{i+1}^{x}+\sigma_{i}^{y} \sigma_{i+1}^{y}+\delta \sigma_{i}^{z} \sigma_{i+1}^{z}\right)\right],
$$

with $g_{i}$ and $h_{i}$ determined by the classical minimum equilibrium configurations of the Hamiltonian, $W_{i}=0$. Note that the external magnetic field depends on the asymmetry parameter $\delta$. This will lead to important consequences on the phase structure of the spin Hamiltonian. We would like to point out here that we have chosen a particular deformation of $H$ for our discussions. One can choose to work with several other deformations of the Hamiltonian $H$. A deformed-Hamiltonian different from (40) has been presented in Appendix D describing Trigonometric model. We use the fermionic representation (40) for our discussions from now onwards. 
Note that $\phi_{0}$ given in Eq. (6) is an exact eigenstate of $\mathcal{H}$ with zero eigenvalue. Now recall that $H$ is bounded from below owing to supersymmetry and the fermionic quartic term in $\mathcal{H}$ is semi-positive. Thus, this is indeed the exact groundstate of $\mathcal{H}$ with the constraints $\delta h_{i} \geq 0$ for all $i$. The constraints for our choices of $W$ for the super-model discussed before imply a relation involving the deformation-parameter $\delta$ and the many-body coupling of the super-model. We now specialize to the rational $W$ only, since it has many advantages over the Trigonometric and Hyperbolic models. The latter models are discussed in Appendices D and E, respectively.

The supersymmetry is lost when $\delta \neq 0$, in terms of the usual realization (1) of the supercharge. However, the supersymmetry can be realized for the rational super-model $\mathcal{H}$ in terms of some new, non-standard representation of the supercharge. We discuss here only the rational $W$ of $A_{N+1}$ type. However, we should note that this is true for any $W$ satisfying (13) and (14). Let us choose $g_{i}$ and $h_{i}$ such as given in (16). The Hamiltonian $\mathcal{H}_{R}$ which generalizes $H_{R}$ of (17) now reads,

$$
\begin{aligned}
\mathcal{H}_{R} & =-\frac{1}{2} \sum_{i} \frac{\partial^{2}}{\partial x_{i}^{2}}+\frac{\lambda^{2}}{2} \sum_{i}\left[2\left(x_{i}-x_{i+1}\right)^{-2}-\left(x_{i-1}-x_{i}\right)^{-1}\left(x_{i}-x_{i+1}\right)^{-1}\right]+\frac{1}{2} \omega^{2} \sum_{i} x_{i}^{2} \\
& +\lambda \sum_{i}\left[\left(x_{i}-x_{i+1}\right)^{-2}\left(\frac{1}{2}\left(n_{i}+n_{i+1}\right)-\psi_{i}^{\dagger} \psi_{i+1}+\psi_{i} \psi_{i+1}^{\dagger}-2 \delta \psi_{i}^{\dagger} \psi_{i} \psi_{i+1}^{\dagger} \psi_{i+1}\right)+\frac{\omega}{2 \lambda} n_{i}\right] \\
& -\lambda \omega N .
\end{aligned}
$$

Note that the wave-function given in (18) still gives the ground-state of $\mathcal{H}_{R}$ with zero energy for $\lambda>0, \delta<0$. This is, in fact, the groundstate for arbitrary $\delta$. In order to see this, we note that the bosonic potential appearing in the above Hamiltonian, except for the confining harmonic term, is a homogeneous function of the bosonic coordinates with degree -2. Following Ref. [9], the Hamiltonian can be transformed to a free super-oscillators through a similarity transformation,

$$
H_{\text {sho }}=T_{1} \mathcal{H} T_{1}^{-1}
$$


where $W$ in $T_{1}=e^{\frac{S_{1}}{2}} e^{W}$ is given by (15) and $S_{1}$ is determined by,

$$
S_{1}=S+2 \delta \lambda \sum_{i}\left(x_{i}-x_{i+1}\right)^{-2} \psi_{i}^{\dagger} \psi_{i} \psi_{i+1}^{\dagger} \psi_{i+1}
$$

Here we recall that $S$ has been defined by (21). Thus, the deformed-Hamiltonian $\mathcal{H}_{R}$ is mapped to a set of free superoscillators. Now we define the odd operator $q$ and its conjugate $q^{\dagger}$ as,

$$
q=T_{1}^{-1} \sum_{i} \psi_{i}^{\dagger} b_{i}^{-} T_{1}, \quad q^{\dagger}=T_{1}^{-1} \sum_{i} \psi_{i} b_{i}^{+} T_{1}
$$

These two operators and the Hamiltonian $\mathcal{H}_{R}$ satisfy the superalgebra. Therefore $\mathcal{H}_{R}$ is bounded from below and the wavefunction given in (18) is its exact groundstate for arbitrary $\delta$. We have found a new, non-standard realization of supercharge giving rise to a quantum mechanical super-Hamiltonian. We have used the term non-standard in the sense that the explicit forms of $q$ and $q^{\dagger}$ involve higher order derivatives and also depends on the fermionic variables in a non-linear way.

The Hamiltonian $\mathcal{H}_{R}$ has dynamical $O(2,1) \times U(1)$ symmetry. The radial excitations can be calculated easily by acting different powers of the creation operator $\mathcal{B}_{2}^{+}$, defined in Appendix $\mathrm{F}$ with $\mathcal{B}_{0}$ replaced by $\mathcal{B}_{0}-\lambda \delta \sum_{i}\left(x_{i}-x_{i+1}\right)^{-2} \psi_{i}^{\dagger} \psi_{i} \psi_{i+1}^{\dagger} \psi_{i+1}$, on $\phi_{0}$. Since we have shown that $\mathcal{H}_{R}$ can be mapped to a set of free superoscillators through the similarity transformation, the problem now reduces to construct the eigenfunctions of $\mathcal{H}_{R}$ from suitable free super-oscillator basis. We find the following $N_{f}=1$ solution of $\mathcal{H}_{R}$,

$$
\chi=T_{1}^{-1} \sum_{i} x_{i}^{n} \psi_{i}^{\dagger} \mid 0>, \quad n=0,1,2,3
$$

We are not able to find any other physically acceptable eigenstates.

Before ending this section, we just mention about the applicability of this technique to other interacting fermionic theories. We recall at this point that the Hubbard model can be viewed as an interacting theory of two different XY models. Let us denote the Hamiltonian 
in (11) as $H^{\psi}$, where the superscript implies that the fermionic variables in $H$ are denoted by $\psi=\left\{\psi_{1}, \psi_{2}, \ldots, \psi_{N}\right\}$. Now define a new Hamiltonian $\tilde{H}$ as,

$$
\tilde{H}=\frac{1}{2}\left(H^{\psi}+H^{\tilde{\psi}}\right)+\delta \sum_{i} h_{i} \psi_{i}^{\dagger} \psi_{i} \tilde{\psi}_{i}^{\dagger} \tilde{\psi}_{i}
$$

We fix the convention that $\psi_{i}$ denotes the spin-up component, while the $\tilde{\psi}_{i}$ denotes the spindown component of the same $i$ th particle. With this identification in mind, it is easy to see that the Hubbard model is contained in the Hamiltonian (48). In the freezing limit, $W_{i}=0$, this Hubbard Hamiltonian can be decoupled from the parent Hamiltonian. Due to the presence of the $O(2,1)$ symmetry in the rational case, an infinite number of exact eigenstates corresponding to the radial excitations can be calculated easily. Also following Ref. [9], $\tilde{H}$ can be mapped to a much simpler Hamiltonian through a similarity transformation for the choice of rational potential. In particular,

$$
\begin{aligned}
\tilde{H}_{\text {sho }} & =H_{\text {sho }}+\omega \sum_{i} \tilde{\psi}_{i}^{\dagger} \tilde{\psi}_{i} \\
& =\tilde{T}^{-1} \tilde{H} \tilde{T},
\end{aligned}
$$

where $\tilde{T}=e^{\frac{\tilde{S}}{2}} e^{W}$. The $W$ in $\tilde{T}$ is given by (15), while $\tilde{S}$ is determined by,

$$
\tilde{S}=\frac{1}{2}\left(S^{\psi}+S^{\tilde{\psi}}\right)+\delta \sum_{i} h_{i} \psi_{i}^{\dagger} \psi_{i} \tilde{\psi}_{i}^{\dagger} \tilde{\psi}_{i}
$$

We would like to point out that one can in fact choose,

$$
G=\prod_{i=1}^{N} \prod_{k=1}^{r}\left(x_{i}-x_{i+k}\right)^{\lambda}
$$

to produce an interacting fermionic theory with $r$ th neighbor interaction in the FL. A suitable deformation of such theories will produce Hubbard model with $r$ th neighbor interaction. However, finding the exact eigenstates, other than those infinitely many radial excitations due to the underlying $O(2,1)$ symmetry, is a nontrivial task and left for a future investigation. 


\section{SUMMARY AND DISCUSSIONS}

We have constructed and studied the supersymmetric version of a class of many-particle Hamiltonians related to the short-range Dyson model. The fermionic sector of these Hamiltonians contains inhomogeneous XY models in an external magnetic field. We have shown that there exists a natural FL for any super-model with classically stable minimum equilibrium configurations, in which the fermionic degrees of freedom can be decoupled completely from the kinematical one. In the FL, the XY model decouples completely from the parent super-model. This implies that the exact eigen-spectrum of the XY spin chain can be constructed from the corresponding states of the full super-model.

We have shown that the rational super-model for both the cases of $A_{N+1}$ and $B C_{N+1}$ can be mapped to the set of free superoscillators through the similarity transformation. This mapping is very useful for constructing the exact eigenstates of the Hamiltonian, which should be otherwise very difficult and intractable. We have constructed many exact eigenstates of the super-model using this mapping. For the $A_{N+1}$-type rational super-model, the spectrum is that of three free superoscillators with the frequencies $\omega, 2 \omega, 3 \omega$ and one

fermionic oscillator with the frequency $4 \omega$. It appears that the spectrum is not complete and also the disparity between the bosonic and the fermionic excitations in the spectrum is not clear at this point. The exact eigen-states corresponding to this spectrum are obtained from the free superoscillator basis which are invariant under the combined exchange of the bosonic and the fermionic coordinates. Though there are no compelling reasons to choose only such symmetric basis, our effort to construct a well-behaved eigen-function of the super-model from a superoscillator basis without this permutation symmetry has failed.

For the $B C_{N+1}$-type super-model, the spectrum is that of a superoscillator with the frequency $2 \omega$ and a fermionic oscillator with the frequency $4 \omega$. The disparity between the 
bosonic and the fermionic excitations are seen again in this case as in its $A_{N+1}$ counterpart. It seems that the spectrum is not complete. The exact eigenstates corresponding to the exact spectrum are obtained from the free superoscillator basis which are invariant under, (i) the combined exchange of the bosonic and the fermionic coordinates and (ii) the simultaneous reflection of the bosonic and the fermionic coordinates. Again, there are no compelling reasons to consider only such superoscillator basis with the specified permutation and reflection symmetry. Unfortunately, we are unable to find any other basis which produces physically acceptable non-singular wavefunction for the super-model.

We have also constructed the exact ground-state of the Trigonometric and the Hyperbolic models. However, in absence of any mapping as in the case of the rational model, the task of finding the excited states are nontrivial and has not been addressed in this paper.

We have considered a suitable deformation of these super-models such that an inhomogeneous XXZ spin chain can be obtained in the FL. This again opens up an alternative method of studying the XXZ spin chains from super-models. We are able to find the exact groundstate of this deformed super-model for the rational, trigonometric and the hyperbolic case. Finding the exact ferromagnetic groundstate of a manifestly semi-positive Hamiltonian will not surprise anybody. However, we have shown that the rational version of these deformed super-models can be mapped to free superoscillators through the similarity transformation. We have also constructed an exact eigenstate of this rational model with the fermion number one. Unfortunately, we are unable to find any more exact eigenstates of this model. However, we believe that the mapping of the rational model to the free superoscillator will allow us to obtain more exact states in future.

The supersymmetry of the deformed-super-model in terms of the usual realizations of the supercharges is lost. However, for the rational case, this model is mapped to the set of free superoscillators through the similarity transformation. Using this mapping, we have 
shown that the deformed-super-model with the rational potential is also supersymmetric in terms of the new, non-standard realization of the supercharges. It remains to be seen whether such realization of the supercharges in theories with $O(2,1)$ symmetry has any further consequences or not.

It is known that the Haldane-Shastry spin chains [13] can be obtained from the CMS model with internal degrees of freedom in the FL [4]. It is also known that the superCMS model describes a particular sector of the usual CMS model with internal $S U(N)$ degrees of freedom [5, 10]. Thus, in the FL, the super-CMS model describes only a particular sector of the Haldane-Shastry spin chains. On the other hand, the super-model considered in this paper describes the full sector of different NN XY models in the FL. Further, the deformed-super-models describe the full sector of different NN XXZ models in the same limit. Unfortunately, at present, we are unable to ascertain the exact solvability of these parent super-models or the deformed-super-models. It might be recalled here that the superpotential $W$ with the constraints (10), (13) and (14) produces (deformed-)super-models, (i) which can be mapped to free superoscillators through the similarity transformation and (ii) give rise to various spin chain Hamiltonians in the FL. Even with all these constraints, there are many choices for $W$. It would be nice if at least one $W$ can be chosen such that the corresponding (deformed-)super-model is exactly solvable.

\section{ACKNOWLEDGMENTS}

We would like to thank Avinash Khare for a careful reading of the manuscript and valuable comments. The work of PKG is supported by a fellowship (P99231) of the Japan Society for the Promotion of Science. This work is partially supported by the Grant-in-Aid for encouragement of Young Scientists (No. 12740231). 


\section{APPENDIX A: JORDAN-WIGNER TRANSFORMATION}

We reproduce the results concerning the Jordan-Wigner transformation in this Appendix. The XXZ Hamiltonian is given by,

$$
H_{x x z}=\frac{1}{2} \sum_{j}\left(\sigma_{j}^{x} \sigma_{j+1}^{x}+\sigma_{j}^{y} \sigma_{j+1}^{y}\right)+\frac{\delta}{2} \sum_{j} \sigma_{j}^{z} \sigma_{j+1}^{z},
$$

where $\sigma$ 's are the Pauli matrices and $\delta$ is the asymmetry parameter. The XY model can be obtained by simply putting $\delta=0$. The above Hamiltonian can be rewritten as,

$$
H_{x x z}=\sum_{j}\left[\left(\sigma_{j}^{+} \sigma_{j+1}^{-}+\sigma_{j}^{-} \sigma_{j+1}^{+}\right)+\frac{\delta}{2} \sigma_{j}^{z} \sigma_{j+1}^{z}\right], \quad \sigma_{j}^{ \pm}=\frac{1}{2}\left(\sigma_{j}^{x} \pm i \sigma_{j}^{y}\right) .
$$

We define the Jordan-Wigner transformation which transforms the spin variables $\sigma_{i}$ 's to the fermionic variables $\psi_{i}$ 's,

$$
\psi_{j}=e^{i \pi \sum_{k=1}^{j-1} \sigma_{k}^{+} \sigma_{k}^{-}} \sigma_{j}^{-}, \quad \psi_{j}^{\dagger}=e^{-i \pi \sum_{k=1}^{j-1} \sigma_{k}^{+} \sigma_{k}^{-}} \sigma_{j}^{+}
$$

with,

$$
\sigma_{i}^{+} \sigma_{i+1}^{-}=\psi_{i}^{\dagger} \psi_{i+1}, \quad \sigma_{i}^{-} \sigma_{i+1}^{+}=-\psi_{i} \psi_{i+1}^{\dagger}, \quad \psi_{i}^{\dagger} \psi_{i}=\sigma_{i}^{+} \sigma_{i}^{-}=\frac{1}{2}\left(1+\sigma_{i}^{z}\right)
$$

The inverse of (A3) is given by,

$$
\sigma_{j}^{-}=e^{i \pi \sum_{k=1}^{j-1} \psi_{k}^{\dagger} \psi_{k}} \psi_{j}, \quad \sigma_{j}^{+}=e^{-i \pi \sum_{k=1}^{j-1} \psi_{k}^{\dagger} \psi_{k}} \psi_{j}^{\dagger}
$$

Note that the fermionic variables satisfy the Clifford algebra (2). The Hamiltonian $H_{x x z}$ in (A1) with periodic boundary condition now reads as,

$$
H_{x x z}=\sum_{i}\left(\psi_{i}^{\dagger} \psi_{i+1}-\psi_{i} \psi_{i+1}^{\dagger}\right)+\frac{\delta}{2} \sum_{i} n_{i} n_{i+1}
$$

We get different types of fermionic Hamiltonians in the FL of the super-models, which have similarity with the above Hamiltonian. The use of the transformations (A3), (A4) and (A5) will enable us to write the corresponding spin Hamiltonian. 


\section{APPENDIX B: QUADRATIC SUPERPOTENTIAL AND XY MODEL}

In this appendix, we discuss about supersymmetric model with the superpotential being quadratic in the bosonic coordinates. The bosonic and the fermionic degrees of freedom decouple automatically for this kind of superpotentials. We further restrict the superpotential to be of a particular form such that the fermionic part describes an inhomogeneous XY model in an external non-uniform magnetic field. Let us choose the following form of the superpotential,

$$
W=\frac{1}{2} \sum_{i} a_{i} x_{i}^{2}+\sum_{i} b_{i} x_{i} x_{i+1}, x_{N+i}=x_{i}
$$

where $a_{i}$ 's and $b_{i}$ 's are arbitrary constants. This amounts to choosing $g_{i}=a_{i}$ and $h_{i}=b_{i}$ in equations (10), (11) and (12). The Hamiltonian $H_{s}$ (fermionic part of $H$ ) completely decouples from the super-Hamiltonian $H$ and describes the most general inhomogeneous isotropic XY model in a non-uniform external magnetic field,

$$
H_{s}=\frac{1}{2} \sum_{i}\left[a_{i} \sigma_{i}^{z}+b_{i}\left(\sigma_{i}^{x} \sigma_{i+1}^{x}+\sigma_{i}^{y} \sigma_{i+1}^{y}\right)\right] .
$$

The XY model as the fermionic part of a supersymmetric quantum mechanics for the particular choice of the superpotential (B1]) with $a_{i}=a$ and $b_{i}=b$ for all $i$ has first been noticed in 23. Later, the same model has been studied from the point of view of supersymmetry breaking in quantum mechanical models [24] with finite degrees of freedom.

The techniques of supersymmetry are not much useful in studying the spectrum of $H_{s}$, since it is completely decoupled from the super-Hamiltonian $H$. Diagonalising the Hamiltonian $H$ essentially implies the diagonalisation of $H_{k}$ ( bosonic part of $H$ ) and $H_{s}$ separately or vice versa. However, we notice an interesting relation between the supersymmetric phase of $H$ and the ferromagnetic phase of $H_{s}$. In particular, the groundstate of $H$ with zero eigen-value is given by, 


$$
\phi_{0}=e^{-\left(\frac{1}{2} \sum_{i} a_{i} x_{i}^{2}+\sum_{i} b_{i} x_{i} x_{i+1}\right)} \mid 0>
$$

The fermionic vacuum $\mid 0>$ in $\phi_{0}$ describes the completely ferromagnetic state in terms of spin degrees of freedom. The normalizability of $\phi_{0}$ demands,

$$
b_{i}^{2} \leq a_{i} a_{i+1}, i=1,2, \ldots N-1, N>5,
$$

with each $a_{i}$ being positive. There are additional relations involving $b_{N}$ for $N<5$. Thus, $\phi_{0}$

with the constraints (B4) is the supersymmetric groundstate of $H$. The supersymmetry is broken when the relations ( $(\mathbb{B} 4)$ are not satisfied, and the groundstate energy of the Hamiltonian $H$ will be positive definite with some well-behaved eigenfunctions. We now notice that the groundstate of $H_{s}$ is given by the fermionic vacuum or the completely ferromagnetic state, only in the case when the relation (B4) is satisfied. Thus, there is a one to one correspondence between the supersymmetric phase of $H$ and the ferromagnetic phase of $H_{s}$. We now discuss a particular example of a homogeneous isotropic XY model in an external staggered magnetic field by choosing,

$$
a_{i}=m+(-1)^{i} m_{s}, \quad b_{i}=b .
$$

We find from Eq. (B4) that the supersymmetric phase and, hence, the ferromagnetic phase is described in the region $m>\left(b^{2}+m_{s}^{2}\right)^{\frac{1}{2}}$ of the parameter space. It is known [25] that the anti-ferromagnetic to ferromagnetic transition of $H_{s}$, with the choice of $a_{i}$ and $b_{i}$ given by (B5), occurs exactly at this critical value.

\section{APPENDIX C: CLASSICAL EQUILIBRIUM CONFIGURATIONS}

The classical equilibrium configuration of $H$ is determined by Eq. (29). In terms of rescaled variables $x_{i} \rightarrow \sqrt{\omega} x_{i}$, this reads, 


$$
x_{i}+\left[\left(x_{i-1}-x_{i}\right)^{-1}-\left(x_{i}-x_{i+1}\right)^{-1}\right]=0
$$

It is trivial to solve the above equations for $N=2$, giving $x_{1}=-1, x_{2}=1$ or vice-versa. For $\mathrm{N}=3$, the zeroes of the third Hermite polynomial satisfy the above equations. We have the following solution for $N=4$,

$$
x_{1}=0, x_{2}=-\sqrt{2}, x_{3}=0, x_{4}=\sqrt{2}
$$

Note that although the coordinates $x_{1}$ and $x_{3}$ are identical, it does not produces any singularity because the interaction depends on the distance between two NN particles only. We do not know any solution for $N \geq 5$. The equation (C1) for arbitrary $N$ can be rewritten as,

$$
\eta_{i-1} \eta_{i+1}\left(\eta_{i}^{2}-2\right)+\eta_{i}\left(\eta_{i-1}+\eta_{i+1}\right)=0, \quad \eta_{i}=x_{i}-x_{i+1}
$$

Now note that $\eta_{i}=\eta$ for all $i$ is not a solution of the above equation. Thus, the equilibrium configurations do not constitute an uniform lattice. In passing, we also note the following identities for the equilibrium configuration,

$$
\sum_{i} x_{i}=0, \sum_{i} x_{i}^{2}=N, \sum_{i} x_{i}^{3}=0 .
$$

Though it would be nice to obtain such identities for arbitrary powers, as in the case of usual CMS, we are unable to find them.

The classical equilibrium configuration of the $B C_{N+1}$-type rational model in terms of the rescaled variables $x_{i} \rightarrow \sqrt{\frac{\omega}{\lambda}} x_{i}$ reads as,

$$
x_{i}+2 x_{i}\left[\frac{1}{x_{i-1}^{2}-x_{i}^{2}}-\frac{1}{x_{i}^{2}-x_{i+1}^{2}}\right]-\frac{\lambda_{1}}{\lambda x_{i}}=0 .
$$

One can check again that the uniform lattice is not a solution of this equation. Note the identity,

$$
\sum_{i} x_{i}^{2}=\left(2+\frac{\lambda_{1}}{\lambda}\right) N
$$

for the equilibrium configurations. 


\section{APPENDIX D: TRIGONOMETRIC MODEL}

We choose the superpotential as,

$$
W=-\ln \prod_{i} \sin ^{\beta}\left[\frac{\pi}{L}\left(x_{i}-x_{i+1}\right)\right], x_{N+i}=x_{i}
$$

This produces the following expressions for $g_{i}$ and $h_{i}$,

$$
h_{i}=-\frac{\beta \pi}{L} \sin ^{-2}\left[\frac{\pi}{L}\left(x_{i}-x_{i+1}\right)\right], \quad g_{i}=-\left(h_{i}+h_{i-1}\right),
$$

and the Hamiltonian $H=H_{k}+H_{s}$ now reads as,

$$
\begin{aligned}
& H_{s}=\frac{\beta \pi^{2}}{2 L^{2}} \sum_{i} \sin ^{-2} q_{i}\left(n_{i}+n_{i+1}-2\left(\psi_{i}^{\dagger} \psi_{i+1}-\psi_{i} \psi_{i+1}^{\dagger}\right)\right) \\
& H_{k}=\frac{1}{2} \sum_{i} p_{i}^{2}+\frac{\beta^{2} \pi^{2}}{L^{2}} \sum_{i}\left(\sin ^{-2} q_{i}-\cot q_{i-1} \operatorname{ccot} q_{i}-1\right), \quad q_{i}=\frac{\pi}{L}\left(x_{i}-x_{i+1}\right) .
\end{aligned}
$$

The supersymmetric groundstate of $H$ for $\beta>0$ is obtained as,

$$
\psi_{0}^{+}=\prod_{i} \sin ^{\beta} q_{i} \mid 0>
$$

On the other hand, for $\beta<0$, the supersymmetric groundstate is given by,

$$
\psi_{0}^{-}=\prod_{i} \sin ^{-\beta} \mid \overline{0}>
$$

The groundstate $\psi_{0}^{+}$is in the zero fermion sector, while it is in the $N$ fermion sector for $\psi_{0}^{-}$. It is amusing to note, unlike in the rational case, the supersymmetric phase is obtained for $\beta>0$ as well as for $\beta<0$. The Trigonometric model is defined on a circle and there is no need to add extra confining potential to stabilize the system. This difference with the rational case is manifested in determining the supersymmetry-preserving and supersymmetry-breaking phases of the two models.

Consider a new Hamiltonian $\mathcal{H}$

$$
\mathcal{H}=H+\frac{\beta \pi^{2} \delta}{2 L^{2}} \sum_{i} \sin ^{-2} q_{i}\left(1-n_{i} n_{i+1}\right)
$$


Both $\psi_{0}^{+}$and $\psi_{0}^{-}$are valid eigenstates of $\mathcal{H}$ with zero eigenvalue for $\beta>0$ and $\beta<0$, respectively. The super-Hamiltonian $H$ is bounded from below, $H \geq 0$, due to the supersymmetry. Now note the following identity for each index $i$,

$$
\left(1-n_{i} n_{i+1}\right)=\frac{1}{2}\left(n_{i}-n_{i+1}\right)^{2} \geq 0
$$

As a consequence, the Hamiltonian $\mathcal{H}$ is also bounded from below for (i) $\beta>0, \delta>0$ and (ii) $\beta<0, \delta<0$. Thus, both $\psi_{0}^{+}$and $\psi_{0}^{-}$are in fact the groundstates of $\mathcal{H}$ in the region (i) and (ii) of the parameter space, respectively.

In the freezing limit, the XY model and the XXZ model can be obtained from $H$ and $\mathcal{H}$, respectively. In this limit, the bosonic coordinates in $H_{s}$ and $\mathcal{H}_{s}$ are determined by the equation,

$$
W_{i}=\frac{\beta \pi}{L}\left[\cot q_{i-1}-\cot q_{i}\right]=0
$$

This equation has the solution that all the particles are equispaced on a circle with the angular separation between two particles given by, $\frac{2 \pi}{N}$. Thus, the corresponding spin chain Hamiltonians are homogeneous XY and XXZ models in an external uniform magnetic field. These models are in fact exactly solvable. The ground state of the XY model is massless and located at the boundary of the ferromagnetic gapped phase. On the other hand, the ground state of the XXZ model is massive and it is in the ferromagnetic gapped phase.

\section{APPENDIX E: HYPERBOLIC MODEL}

We choose the superpotential as,

$$
W=-\ln \prod_{i} \sinh ^{\beta}\left(x_{i}-x_{i+1}\right)+\gamma \sum_{i} e^{2 x_{i}}, x_{N+i}=x_{i}
$$


The first term of (E1) can be obtained from (D1) by rescaling, $x_{j} \rightarrow \frac{L}{\pi} i x_{j}$, where $i$ is the imaginary number. The second term has been introduced to stabilize the system. It produces a confining Morse potential. The expression for $g_{i}$ and $h_{i}$ are,

$$
h_{i}=\beta \sinh ^{-2} q_{i}, \quad g_{i}=-\left(h_{i}+h_{i-1}\right)+4 \gamma e^{2 x_{i}}, \quad q_{i}=x_{i}-x_{i+1},
$$

with the Hamiltonian $H=H_{k}+H_{s}$ given by,

$$
\begin{aligned}
& H_{k}=\sum_{i}\left[\frac{p_{i}^{2}}{2}+\beta^{2}\left(\sinh ^{-2} q_{i}-\operatorname{coth} q_{i-1} \operatorname{coth} q_{i}+1\right)+2 \gamma^{2} e^{4 x_{i}}-4 \gamma \beta e^{2 x_{i}}\right] \\
& H_{s}=\frac{\beta}{2} \sum_{i}\left[-\sinh ^{-2} q_{i}\left\{n_{i}+n_{i+1}-2\left(\psi_{i}^{\dagger} \psi_{i+1}-\psi_{i} \psi_{i+1}^{\dagger}\right)\right\}+\frac{4 \gamma}{\beta} e^{2 x_{i}} n_{i}\right]
\end{aligned}
$$

Note that the bosonic sub-model of $H$ ( i.e. the zero-fermion sector ) is the nearestneighbor variant of the corresponding CMS Hamiltonian [26]. The ground-state of $H$ in the supersymmetry-preserving phase $(\beta>0, \gamma>0)$ is given by,

$$
\psi_{0}=\prod_{i} \sinh ^{\beta} q_{i} e^{-\gamma \sum_{i} e^{2 x_{i}}} \mid 0>
$$

This is also the groundstate of,

$$
\mathcal{H}=H+\frac{\beta \delta^{2}}{2} \sum_{i} \sinh ^{-2} q_{i} \psi_{i}^{\dagger} \psi_{i} \psi_{i+1}^{\dagger} \psi_{i+1},
$$

with zero eigenvalue. We do not know the groundstates of $H$ and $\mathcal{H}$ in other regions of the parameter space of $\beta$ and $\gamma$. This is because the 'method of duality' used for the rational case does not work for the specific confining potential of this Hyperbolic model. In the FL, the Hamiltonian $H$ and $\mathcal{H}$ gives rise to XY and XXZ Hamiltonian, respectively. In this limit, the bosonic coordinates in $H_{s}$ and $\mathcal{H}_{s}$ are determined by the following relation,

$$
W_{i}=\beta\left(\operatorname{coth} p_{i-1}-\operatorname{coth} p_{i}\right)+2 \gamma e^{2 x_{i}}=0 .
$$

We do not know the solution of this equation. 


\section{APPENDIX F: $O S P(2 \mid 2)$ SUPER-ALGEBRA}

We show in this appendix that the Hamiltonian $H$ in (8), with the superpotential given by Eq. (13) and (14), has dynamical $O S p(2 \mid 2)$ supersymmetry. We work with $\omega=1$ in this Appendix without loss of any generality. We first define the following four supercharges,

$$
\begin{aligned}
& q=\frac{1}{2} \sum_{i=1}^{N} \psi_{i}^{\dagger}\left(p_{i}+i w_{i}-i x_{i}\right), \quad q^{\dagger}=\frac{1}{2} \sum_{i=1}^{N} \psi_{i}\left(p_{i}-i w_{i}+i x_{i}\right), \\
& \tilde{q}=\frac{1}{2} \sum_{i=1}^{N} \psi_{i}\left(p_{i}-i w_{i}-i x_{i}\right), \quad \tilde{q}^{\dagger}=\frac{1}{2} \sum_{i=1}^{N} \psi_{i}^{\dagger}\left(p_{i}+i w_{i}+i x_{i}\right)
\end{aligned}
$$

The Hamiltonian $H$ is given in terms of $q$ and $q^{\dagger}$ as, $H=2\left\{q, q^{\dagger}\right\}$. The dual Hamiltonian $\mathcal{H}^{d}$ can be constructed in terms of $\tilde{q}$ and $\tilde{q}^{\dagger}, \mathcal{H}^{d}=2\left\{\tilde{q}, \tilde{q}^{\dagger}\right\}$. We define the following operators,

$$
\begin{aligned}
& h=\frac{1}{2}\left(H+\mathcal{H}^{d}\right), \quad U=\frac{1}{2}\left(H-\mathcal{H}^{d}\right), \quad E=\sum_{i} x_{i} \frac{\partial}{\partial x_{i}}, \\
& \mathcal{B}_{2}^{-}=\mathcal{B}_{0}-\frac{1}{4} \sum_{i} x_{i}^{2}-\frac{1}{4}(N+2 E), \quad \mathcal{B}_{2}^{+}=\mathcal{B}_{0}-\frac{1}{4} \sum_{i} x_{i}^{2}+\frac{1}{4}(N+2 E), \\
& \mathcal{B}_{0}=\frac{1}{4} \sum_{i}\left(p_{i}^{2}+w_{i}^{2}+w_{i i}-2 \sum_{j} w_{i j} \psi_{i}^{\dagger} \psi_{j}\right) .
\end{aligned}
$$

The bosonic operators $\mathcal{B}_{2}^{ \pm}$and $h$ satisfies the following relations,

$$
\left[h, \mathcal{B}_{2}^{ \pm}\right]= \pm 2 \mathcal{B}_{2}^{ \pm}, \quad\left[\mathcal{B}_{2}^{-}, \mathcal{B}_{2}^{+}\right]=h
$$

The commutator relation $\left[E, \mathcal{B}_{0}\right]=-2 \mathcal{B}_{0}$ has been used in deriving the above equations. The $U(1)$ generator $U$ commutes with $\mathcal{B}_{2}^{ \pm}$and $h$.

The non-vanishing anticommutators among $q, q^{\dagger}, \tilde{q}$ and $\tilde{q}^{\dagger}$ are,

$$
\left\{q, q^{\dagger}\right\}=\frac{1}{2}(h+U), \quad\left\{\tilde{q}, \tilde{q}^{\dagger}\right\}=\frac{1}{2}(h-U), \quad\left\{q^{\dagger}, \tilde{q}^{\dagger}\right\}=\mathcal{B}_{2}^{+}, \quad\{q, \tilde{q}\}=\mathcal{B}_{2}^{-}
$$

Observe that the relation,

$$
H=\mathcal{H}_{d}+2 U,
$$


which is useful in determining the spectrum in the supersymmetry breaking phase, follows easily from the first two equations of ([F4).

The other non-vanishing commutators are,

$$
\begin{aligned}
& {\left[\mathcal{B}_{2}^{+}, q\right]=-\tilde{q}^{\dagger}, \quad\left[\mathcal{B}_{2}^{+}, \tilde{q}\right]=-q^{\dagger}, \quad\left[\mathcal{B}_{2}^{-}, \tilde{q}^{\dagger}\right]=q, \quad\left[\mathcal{B}_{2}^{-}, q^{\dagger}\right]=\tilde{q},} \\
& {\left[h, q^{\dagger}\right]=q^{\dagger}, \quad[h, q]=-q, \quad[h, \tilde{q}]=-\tilde{q}, \quad\left[h, \tilde{q}^{\dagger}\right]=\tilde{q}^{\dagger},} \\
& {[U, \tilde{q}]=-\tilde{q}, \quad\left[U, \tilde{q}^{\dagger}\right]=\tilde{q}^{\dagger}, \quad\left[U, q^{\dagger}\right]=-q^{\dagger}, \quad[U, q]=q .}
\end{aligned}
$$




\section{REFERENCES}

[1] F. Calogero, J. Math. Phys. (N.Y.) 10 (1969) 2191; 10 (1969) 2197.

[2] B. Sutherland, J. Math. Phys.(N.Y.) 12 (1971) 246; 12 (1971) 251; Phys. Rev. A 4 (1971) 2019.

[3] M. A. Olshanetsky and A. M. Perelomov, Phys. Rep. 71 (1981) 314; 94 (1983) 6.

[4] A. P. Polychronakos, Phys. Rev. Lett. 70 (1993) 2329; A. Polychronakos, Les Houches Lectures 1998, hep-th/9902157.

[5] L. Brink, T. H. Hansson, S. Konstein and M. A. Vasiliev, Nucl. Phys. B401 (1993) 591.

[6] E. D'Hoker and D. H. Phong, hep-th/9912271; A. Gorsky and A. Mironov, hepth/0011197.

[7] A. J. Bordner, E. Corrigan and R. Sasaki, Prog. Theor. Phys. 102 (1999) 499, hepth/9905011; A. J. Bordner, E. Corrigan and R. Sasaki, Prog. Theor. Phys. 100 (1998) 1107, hep-th/9805106; A. J. Bordner, R. Sasaki and K. Takasaki, Prog. Theor. Phys. 101 (1999) 487, hep-th/9809068; A. J. Bordner and R. Sasaki, Prog. Theor. Phys. 101 (1999) 799, hep-th/9812232; S. P. Khastgir, R. Sasaki and K. Takasaki, Prog. Theor. Phys. 102 (1999) 749, hep-th/9907102; S. P. Khastgir, A. J. Pocklington and R. Sasaki, J. Phys. A33 (2000) 9033, hep-th/0005277.

[8] N. Gurappa and P. K. Panigrahi, Phys. Rev. B59 (1999) R2490, cond-mat/9710035; quant-phy/9710019; N. Gurappa, A. Khare and P. K. Panigrahi, Phys. Lett. A224 (1998) 467, cond-mat/9804207; K. Sogo, J. Phys. Soc. Jpn. 65 (1996) 3097; H. Ujino, A. Nishino, and M. Wadati, J. Phys. Soc. Jpn. 67 (1998) 2658; T. H. Baker and P. J. Forrester, Nucl. Phys. B492 (1997) 682; T. Brzezinski, C. Gonera and P. Maslanka, 
Phys. Lett. A 254 (1999) 185; B. Basu-Mallick, H. Ujino and M. Wadati, J. Phys. Soc. Jap. 68 (1999) 3219.

[9] P. K. Ghosh, Nucl. Phys. B595 (2001) 519, hep-th/0007208.

[10] D. Z. Freedman and P. F. Mende, Nucl. Phys. B344 (1990) 317; L. Brink, A. Turbiner and N. Wyllard, J. Math. Phys. 39 (1998) 1285; A. J. Bordner, N. S. Manton and R. Sasaki, Prog. Theor. Phys. 103 (2000) 463, hep-th/9910033; N. Wyllard, J. Math. Phys. 41 (2000) 2826, hep-th/9910160; M. V.Ioffe and A. I. Neelov, J. Phys. A33 (2000) 1581, quant-ph/0001063; P. K. Ghosh, J. Phys. A34 (2001) 5583, hep-th/0009055; A. Dabholkar, Nucl. Phys. B368 (1992) 293; J. P. Rodrigues and A. J. van Tonder, Int. J. Mod. Phys. A8 (1993) 2517; B. S. Shastry and B. Sutherland, Phys. Rev. Lett. 70 (1993) 4029.

[11] P. K. Ghosh, A. Khare and M. Sivakumar, Phys. Rev. A58 (1998) 821; C. Efthimiou and D. Spector, Phys. Rev. A56 (1997) 208.

[12] M. V. N. Murthy and R. Shankar, Phys. Rev. Lett. 73 (1994) 3331; Z. N. C. Ha, Phys. Rev. Lett. 73 (1994) 1574; S. B. Isakov, Phys. Rev. Lett. 73 (1994) 2150.

[13] F. D. M. Haldane, Phys. Rev. Lett. 60 (1988) 635; 66 (1991) 1529; B. S. Shastry, Phys. Rev. Lett. 60 (1998) 639; 69 (1992) 164; D. Bernard, V. Pasquier and D. Serban, hepth/9501044; T. Fukui and N. Kawakami, Nucl. Phys. B483 (1997) 663; K. Hikami and B. Basu-Mallick, Nucl. Phys. B566 (2000) 511.

[14] G. W. Gibbons and P. K. Townsend, Phys. Lett. B454 (1999) 187.

[15] F. Dyson, J. Math. Phys. 3 (1962) 140.

[16] O. Bohigas, M. J. Giannoni and C. Schmidt, Phys. Rev. Lett. 52 (1984) 1. 
[17] B. D. Simons, P. A. Lee and B. L. Altshuler, Phys. Rev. Lett. 72 (1994) 64.

[18] S. R. Jain and A. Khare, Phys. Lett. A 262 (1999) 35; G. Auberson, S. R. Jain and A. Khare, J. Phys. A34 (2001) 695, cond-mat/0004012; M. Ezung, N. Gurappa, A. Khare and P. K. Panigrahi, cond-mat/0007005.

[19] B. Grem'and and S. R. Jain, J. Phys. A 31 (1998) L637; A. Pandey, Unpublished; E. Bogomol'ny, U. Gerland and C. Scimt, Phys. Rev. E 59 (1999) R1315; H. D. Parab and S. R. Jain, J. Phys. A29 (1996) 3903.

[20] A. N. Zemlyakov and A. B. Katok, Math. Notes 18 (1976) 760; P. J. Richens and M. V. Berry, Physica D2 (1981) 495; S. R. Jain and H. D. Parab, J. Phys. A 25 (1992) 6669; S. R. Jain and S. V. Lawande, Proc. Indian Natl. Sc. Acad. 61 A (1995) 198.

[21] G. Date, S. R. Jain and M. V. N. Murthy, Phys. Rev. E51 (1995) 198.

[22] T. Guhr, A. Müller- Groeling, H. A. Weidenmüller, Phys. Rep. 299 (1998) 189.

[23] M. de Crombrugghe and V. Rittenberg, Annals of Physics, 151 (1983) 99.

[24] A. Khare and J. Maharana, Nucl. Phys. B244 (1984) 409; A. Khare and J. Maharana, Phys.Lett. B145 (1984) 77.

[25] F. Alcaraz and A. L. Malvezzi, cond-mat/9407050.

[26] H. Frahm and V. I. Inozemtsev, J. Phys. A 27 (1994) L801, cond-mat/9405038. 